\title{
Construcción de un instrumento de verificación de la calidad de portales y redes de investigación de carácter científico en Internet
}

\author{
Ángel De-Juanas*, Rodrigo Pardo**, Alfonso Diestro*, Amelia Ferro**, \\ Javier Sampedro**
}

Resumen: En este estudio se propone un instrumento de verificación de la presencia de indicadores de calidad de portales y web de carácter científico, relacionados con las Ciencias Sociales y de la Salud, que ha sido validado por expertos. Se realizaron tres estudios a partir de un diseño no experimental de tipo mixto: análisis de documentos y valoraciones cualitativas y cuantitativas por parte de jueces. El resultado fue el desarrollo de un instrumento, QuaSciWeb, que contiene 6 categorías de análisis que agrupan 57 ítems: 1) Identidad y autoría; 2) Interfaz del usuario; 3) Contenido; 4) Navegación y recuperación de datos; 5) Experiencia de usuario; 6) visibilidad y divulgación. El instrumento permite: a) Valorar la calidad de portales y páginas web de carácter científico; $b$ ) identificar los contenidos necesarios para su mejora, y $c$ ) proveer de unas directrices concretas que pueden ser usadas por los programadores y diseñadores web.

Palabras clave: Evaluación, calidad, indicadores, web, portales científicos, ciencias sociales, ciencias de la salud, instrumento de verificación, investigación.

\section{Construction of a quality verification tool for Internet-based scientific research portals and networks}

Abstract: This study proposes a verification tool, validated by experts, for detecting the presence of quality indicators in scientific portals and websites related to Social and Health Sciences. Three studies were carried out using a non-experimental mixed design: document analysis and qualitative and quantitative assessments performed by referees. The result was the development of the QuaSciWeb tool which contains 57 items spread over 6 categories of analysis: 1) Identity and authorship; 2) User interface; 3) Contents; 4) Surfing and data recovery; 5) User experience; 6) Visibility and dissemination. The tool permits a) the assessment of the quality of scientific portals and websites, b) the identification of the contents needed for improvement and c) concrete guidelines which can be used by programmers and web designers.

Keywords: Assessment, quality, indicators, web, scientific portals, social sciences, health sciences, verification tool, research.

* Universidad Nacional de Educación a Distancia. Correo-e: adejuanas@edu.uned.es; adiestro@ edu.uned.es.

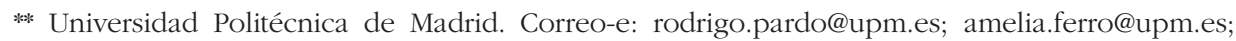
javier.sampedro@upm.es.

Recibido: 20-10-2011; 2. ${ }^{a}$ versión: 17-02-2012; aceptado: 20-02-2012. 


\section{Introducción}

Dada la proliferación de portales y sitios web, se hace cada vez más necesario contar con herramientas que permitan conocer la calidad de los mismos. Tal y como afirman Hasan y Abuelrub (2011): "La explosión de la web ha determinado la necesidad de criterios de medición para evaluar los aspectos relacionados con la calidad en uso, tales como la accesibilidad y usabilidad de una aplicación web» (p. 12).

Para conocer la excelencia de estos portales se han utilizado diferentes técnicas y procedimientos. Por ejemplo, disciplinas como la Cibermetría y la Webmetría surgen a finales de los años noventa para estudiar de forma cuantitativa los contenidos y procesos de comunicación que se producen en el ciberespacio y en la web, respectivamente, a partir de planteamientos bibliométricos e infométricos (Alonso Berrocal y otros, 2008; Arroyo y otros, 2005; Björneborn y Ingwersen, 2001, 2004; Thelwall y otros, 2005).

En concreto, la Webmetría se ocupa del análisis de los enlaces o hipervínculos entre páginas web, citas de publicaciones académicas, evaluación de los motores de búsqueda y estudio descriptivo de páginas web (Thelwall, 2008). Estudios como los realizados por Almind e Ingwersen (1997), Arakaki y Willett (2009), Barjak y Thelwall (2008), Carballo-Pérez y otros (2008), Ortega y Aguillo (2006), Rousseau (1997), Smith (1999), Thelwall y Harries (2003), Thelwall y Sud (2011), y Thomas y Willett (2000), son claros ejemplos.

Mención aparte merece el "Ranking Mundial de Universidades en la web" (www.webometrics.info), elaborado de forma semestral por el Laboratorio de Cibermetría del Consejo Superior de Investigaciones Científicas desde 2004, y que utiliza técnicas de Webmetría para su confección (Marcos, 2006). Entre los estudios que han utilizado esta metodología señalamos los de Aguillo y otros (2006), Ortega y Aguillo (2007, 2009), y Ortega y otros (2006).

Otro aspecto a tener en cuenta es el posicionamiento y visibilidad de una página web (Codina y Marcos, 2005). Quizás sea el PageRank la técnica de mayor éxito (aunque no ajena a cierta controversia) ya que permite valorar la relevancia de una página a través de la notoriedad de las páginas que la enlazan (Page y otros, 1998). En esta misma dirección cabe destacar el Web Impact Factors (Web-if) elaborado por Ingwersen (1998), que consiste en la suma del número de enlaces externos y autoenlaces dirigidos a un país (a través de un dominio, como por ejemplo .es) o página web dividido por el número de páginas encontradas en ese país o página web en un momento concreto. Asimismo hay que destacar el Traffic Rank, que se basa en clasificar las páginas web en función de dos datos: número de visitas y cantidad de páginas visitadas (Alexa Internet, 2011).

Internet también nos ofrece herramientas que permiten determinar el diagnóstico de una página web en cuanto a diferentes aspectos tales como: enlaces recibidos (LexiURL Searcher), accesibilidad (Hera, TAW o Wave), privacidad (WebXact), adaptabilidad para personas con problemas de diferenciación de 
colores (Vischeck), identificar enlaces rotos en el código HTML (Netmechanic) o validación del código HTML (W3C's HTML validator) (Arakaki y Willett, 2009; Choudrie y otros, 2004; Rovira y otros, 2007).

Sin embargo, la tan anhelada "objetividad" parece una difícil tarea a la hora de valorar la calidad de las páginas web, ya que como señalan Olsina y Rossi (2002):

«[...] a veces tenemos que evaluar subjetivamente si los requisitos son satisfechos (como la calidad de las funciones de ayuda o la preferencia estética de un sitio web). Sin embargo, podemos reducir al mínimo la subjetividad en el proceso de evaluación al centrarse en los atributos de manera objetiva, tales como enlaces rotos, páginas huérfanas y rapidez de acceso a las páginas. Una metodología de evaluación sólida y flexible debe incluir ambos componentes subjetivos y objetivos debidamente controlados por expertos.» (p. 28).

\section{Estado de la cuestión}

Existe una gran cantidad de investigaciones dirigidas a valorar la calidad de las páginas web, aunque la mayoría de ellas se centran en el estudio de un tipo de web concreta. Basándonos en la extensa revisión realizada por Hasan y Abuelrub (2011) de los diferentes estudios que han utilizado indicadores de calidad para valorar portales y páginas web, encontramos que éstos pueden dividirse en cuatro grandes grupos:

- Comercio electrónico (Barnes y Vidgen, 2002; Hussin y otros, 2005; Maynard y Tian, 2004; Olsina y Rossi, 2002).

- Educativos/Investigación (Chao, 2002; González-Lucio y otros, 2009; Lautenbach y otros, 2006; Mebrate, 2010; Palatano, 2002; Pinto y otros, 2005; Pinto y otros, 2004; Pinto y otros, 2009; Ribera y otros, 2009; Rovira y otros, 2007).

- Gubernamentales (Batlle-Montserrat y otros, 2011; Choudrie y otros, 2004; Monaghan, 2008; Wyman y otros, 1997).

- Sector bancario (Diniz y otros, 2005; Hussin y otros, 2005; Miranda y otros, 2006; Raman y otros, 2008).

Sin embargo, algunas investigaciones ofrecen indicadores más amplios que evalúan la calidad de cualquier página web independientemente de su temática. Por ejemplo, utilizando la Webmetría en combinación con otras técnicas, nos encontramos con índices de calidad como el WebQual. Este indicador utiliza un total de 22 criterios divididos en tres grandes apartados: usabilidad, calidad de la información y calidad de la interacción con el servicio (Barnes y Vidgen, 2002). Por su parte, Olsina y Rossi (2002) proponen el WebQEM, dirigido a valorar la calidad de las webs de manera cuantitativa en base a más de 90 indicadores que se pueden agrupar en cuatro grandes: apartados: usabilidad, funcionalidad, fiabilidad y eficiencia. Del mismo modo, Hasan y Abuelrub (2011) también estable- 
cen cuatro aspectos a tener en cuenta para determinar la calidad de una página web: contenido, diseño, organización y usabilidad.

En el caso de la presente investigación, resulta necesario distinguir entre las web que tienen un marcado carácter comercial y aquellas dirigidas a aspectos relacionados con la información y la investigación. De ahí que se haya prestado una especial atención a los estudios dirigidos a valorar la calidad de portales y redes de investigación de carácter científico. En este sentido, los estudios más representativos son los relacionados con webs académicas, universidades, bibliotecas y grupos de investigación.

Al respecto, Palatano (2002) analiza las páginas web de 62 bibliotecas universitarias argentinas utilizando un cuestionario con 45 indicadores para establecer la calidad de las mismas. Estos indicadores se agrupan en cuatro grupos: presentación de la información, productos y servicios, información sobre la biblioteca, y conectividad e interacción con el usuario. Asimismo, encontramos otros trabajos como el de González-Lucio y otros (2009) para la evaluación de 68 webs de bibliotecas universitarias españolas analizadas mediante 16 elementos de calidad.

En otro sentido, el estudio de Chao (2002) sobre bibliotecas universitarias de Estados Unidos, reduce este número a 8 indicadores: presentación, contenido, diseño gráfico, compatibilidad, servicios, compatibilidad en la búsqueda, información institucional e información sobre los enlaces. De entre ellos, el autor señala que a la hora de diseñar o evaluar la calidad de una página web hay dos indicadores clave: la riqueza del contenido (eficacia) y su atractivo estructural (eficiencia). Estos mismos términos (eficacia y eficiencia) junto con el de «encontrabilidad" (findability) son los que utilizan Lautenbach y otros (2006) para valorar la usabilidad de una web universitaria. Rovira y otros (2007), utilizando un programa informático de tipo rastreador de desarrollo propio, analizan 230 repositorios de publicaciones digitales de libre acceso utilizando más de 90 indicadores que dividen en tres apartados: accesibilidad, posicionamiento en los buscadores y calidad del código fuente.

Entre los estudios que combinan técnicas cualitativas y cuantitativas, destaca el realizado por Pinto y otros $(2005 ; 2004)$ en el que analizan la calidad de 16 páginas web de universidades españolas. En él se tienen en cuenta 14 indicadores cuantitativos y 58 cualitativos que fueron agrupados en 7 categorías: visibilidad de la información, autoría, actualización y actualidad, accesibilidad, difusión de la investigación, evaluación de la calidad y navegabilidad. Este estudio fue ampliado posteriormente hasta analizar 70 indicadores agrupados en las mismas categorías (Pinto y otros, 2009).

\section{Interés para la comunidad científica}

El interés del trabajo aquí expuesto es el de proponer un cuestionario validado por expertos con un número suficiente y relevante de indicadores a analizar evitando el número excesivo o limitado de otros estudios. Además, este 
cuestionario es específico para portales y páginas web de carácter científico que, aunque ya han sido ampliamente estudiadas a través técnicas Webmétricas, sigue siendo necesario establecer otros tipos de criterios para su valoración ya que la calidad de una página web o de la institución que está detrás de ella no puede ser evaluada exclusivamente por este tipo de técnicas cuantitativas. Por último, los indicadores que aparecen en este cuestionario son perceptibles para el usuario (e.g., interfaz de usuario, contenido, navegación, visibilidad, etc.), sin entrar a analizar aspectos «ocultos» como pueden ser los problemas con el código o la utilización de hojas de estilo.

Por tanto, el presente cuestionario puede ser utilizado con tres objetivos: 1) valorar la calidad de portales y páginas web que ofrezcan contenidos de investigación de carácter científico, 2) identificar los puntos necesarios para su mejora y 3) proveer de unas directrices concretas a tener en cuenta por los programadores y diseñadores web a la hora de construir una página o un portal de estas características.

\section{Método}

\subsection{Diseño}

Para la construcción del instrumento de verificación se realizaron tres tipos de estudio a partir de un diseño no experimental de tipo mixto, puesto que la construcción del instrumento consideró la revisión de la literatura científica afín al objeto de estudio, así como la recogida y el análisis de datos tanto cualitativos como cuantitativos.

\subsection{Procedimiento}

En una primera fase de estudio se especificó la finalidad, propósito y criterio de evaluación del instrumento a construir, así como los aspectos asociados a los posibles indicadores de objeto de valoración. Para ello, se partió de un proceso inicial de validación de contenido en el que se llevó a cabo una revisión bibliográfica conforme al estado de la cuestión: evaluación de la calidad de portales y sitios webs. De tal modo, se seleccionaron más de 90 documentos a partir de la búsqueda en las principales bases de datos (Academic, ERIC, PsycINFO, Information Science, Technology Abstract, Computer Database, ACM Digital Library e ISI Web of Knowledge) y se hizo un análisis de contenido de los mismos. El proceso de búsqueda se llevó a cabo mediante los siguientes términos de consulta: webmetrics analysis; evaluation web site quality; assessment models web sites; web application quality; cibermetría; optimización de sitios web. Los términos utilizados proporcionaron un resultado superior a veinte mil registros. La práctica totalidad de documentos recopilados fueron artículos publicados en re- 
vistas científicas, en su mayoría escritos en lengua inglesa, aunque se incluyeron varios manuscritos en lengua española.

Para la selección de los mismos se utilizó como criterio de relevancia la vinculación de la temática con el propósito de estudio y el número de citas recibidas para cada uno de los registros.

Este proceso de documentación permitió identificar los principales aspectos a tener en consideración como indicadores y elementos de calidad de webs científicas, en el marco de las ciencias sociales y de la salud.

En una segunda fase de estudio, se manejó un amplio volumen de información y se clasificó recogiendo los principales elementos relacionados con los indicadores propuestos. Para ello se creó un banco inicial de ítems siguiendo las directrices expuestas por Muñiz y otros (2005). A saber: definir el objeto de evaluación, crear una tabla de especificación, especificar el contexto y destinatarios, referir cada ítem a un solo elemento de estudio, expresar los ítems en positivo tratando que fueran lo más precisos posibles.

Finalmente, se realizó una última fase de estudio para determinar el instrumento de evaluación a partir del juicio de veinte expertos. De tal modo, en la valoración mediante el juicio de expertos se requirió que los participantes evaluasen los ítems propuestos respecto a los criterios de pertinencia (adecuación de los ítems al objeto de medición dentro de cada uno de los indicadores) y claridad (redacción correcta, claridad y consistencia) propuestos por Millman y Greene (1989). Al mismo tiempo que hicieron una valoración cualitativa sobre los indicadores e ítems.

En relación a la elección de los expertos, en primer lugar se seleccionó a un total de 26 posibles candidatos, fijando como criterio fundamental de elección su competencia en el área de conocimiento en la que se centra esta investigación sobre la base de su currículo personal. Para ello, se les envío por correo postal una carta explicativa sobre los propósitos del estudio junto con el instrumento a evaluar. En la misma se solicitaba su participación y se aseguraba su anonimato. Del total inicial, 20 decidieron participar en el estudio, lo que en base a la literatura consultada aporta la suficiente validez (DeVon y otros, 2007; Lynn, 1986). De esta forma, del total de los jueces participantes, nueve fueron docentes e investigadores pertenecientes a universidades públicas españolas (dos de la Universidad Nacional de Educación a Distancia, uno de la Universidad Complutense de Madrid, uno de la Universidad Autónoma de Madrid y tres de la Universidad Politécnica de Madrid). Todos ellos de diferentes facultades de Ciencias Sociales e Informática que tenían en común su vinculación profesional con las nuevas tecnologías y, en concreto, con el estudio de diferentes páginas web de carácter científico relacionadas con su área de conocimiento. Además, ocho de los jueces fueron informáticos cuya ocupación profesional se centra en el desarrollo de espacios y aplicaciones web. Finalmente, participaron tres empresarios de servicios de carácter científico en Internet.

La función de los expertos fue contribuir a esclarecer la validez de contenido del instrumento. Gracias a sus valoraciones se pudo ajustar la herramienta refi- 
nando el lenguaje y la adecuación de los ítems para cada uno de los indicadores propuestos. Se ha de tener en consideración que sobre la base de la documentación encontrada se establecieron seis grandes indicadores y el criterio de los jueces fue determinante para confirmarlos y agrupar los ítems dentro de cada uno de los mismos.

\subsection{Análisis}

En primer lugar, se realizó un análisis de contenido de los documentos y webs propias a este ámbito. Más adelante, una vez elaborado un primer instrumento piloto que se aplicó a jueces expertos, se generó una base de datos y se procedió a realizar análisis estadísticos mediante el programa informático SPSS versión 19. De tal modo, se calculó la fiabilidad y se realizaron análisis de la validez de contenido mediante el modelo matemático de Lawshe (1975, en Cohen y Swerdlik, 2006). Dicho procedimiento utiliza las opiniones de los jueces con el objetivo de determinar sí cada uno de los ítems era pertinente o no para medir el constructo de los indicadores. De tal modo, se trató de encontrar el valor mínimo de razón de cada ítem, es decir, el coeficiente de validez de razón de contenido $(C V R)$ a partir de las puntuaciones de pertinencia mediante la fórmula:

$$
C V R=\frac{N e-N / 2}{N / 2}
$$

Se trata de un valor promedio en el que $N e$ se corresponde con la cantidad de jueces que valoraron el ítem como pertinente. En nuestro caso, decidimos que un ítem era valorado como pertinente cuando obtenía una valoración igual o mayor a cuatro sobre seis puntos posibles a juicio de cada uno de los expertos. A su vez, $N$ se corresponde con el número total de jueces que participaron en el estudio.

\section{Resultados}

En relación con la primera fase de estudio, tras el análisis de contenido de documentos, se establecieron una serie de indicadores relativos a seis grandes parámetros de interés o categorías de análisis: 1) identidad y autoría; 2) interfaz del usuario; 3) contenido; 4) navegación y recuperación de datos; 5) experiencia de usuario; 6) visibilidad y divulgación.

A continuación se describen los indicadores:

1) Identidad y autoría: hace referencia a los elementos que indican la presencia de datos relativos a la organización, empresa, entidad o institución —o el conjunto de ellas-, que participan, financian o colaboran con el 
sitio web objeto de evaluación. A su vez, es muy importante que los datos de los responsables aparezcan bien descritos — finalidad, objetivos e historia, responsabilidad legal del propietario, etc.-, por cuestiones de transparencia, solvencia y garantía de prestigio. Del mismo modo, la presencia clara y concisa de los ámbitos y contenidos temáticos, así como la magnitud de su contenido, representan una garantía de calidad en la página de inicio (principal escaparate del sitio). También debería ofrecer información precisa para ubicar a los autores, responsables o gestores en su ámbito académico o científico.

2) Interfaz del usuario: la mejor interacción a través de una adecuada interfaz debe ofrecer tanto comodidad, como eficiencia. El principal objetivo de ésta es que el usuario se pueda comunicar e interaccionar con la web. De tal modo, una interfaz de usuario de calidad debe ser funcional y facilitar la interactividad entre los usuarios.

3) Contenidos: una web científica de calidad debe cumplir ciertos requerimientos mínimos que atañen a la información que presenta dado que una información precisa y concreta, otorga una mayor fiabilidad y efectividad de la web.

4) Navegación y recuperación de datos: se refiere a la movilidad, el acceso y la comodidad en la visita a la web, además del soporte de recuperación de la información para el usuario. De tal manera, se considera la importancia de un sistema de búsqueda estructurada, jerarquización de contenidos, navegación intuitiva, entre otros.

5) Experiencia de usuario (look and feel): valora la capacidad de hacer agradable y no fatigosa la visita a los portales y webs por parte de los usuarios. Es lógico señalar que este parámetro constituye un elemento esencial y significativo en cualquier valoración.

6) Visibilidad y divulgación de contenidos: se recogen aquellos elementos que promueven la visibilidad de la web y la divulgación de sus contenidos, a través de enlaces de web, imagen corporativa, etc.

En cuanto a la segunda fase de estudio, conforme al volumen de información encontrada y clasificada, se elaboraron un total de 97 ítems, considerando aquellos que permitían obtener información específica y válida sobre los indicadores presentados. No obstante, el número de elementos creados fueron considerados excesivos, por lo que estos ítems fueron sometidos a un análisis, revisión y reestructuración, por parte de los autores, con el propósito de evitar la reiteración de contenidos. En esta fase se decidieron descartar 27 reactivos, dando lugar a un banco de 70 ítems para ser posteriormente sometidos al criterio de expertos.

Finalmente, debido a la necesidad de garantizar la validez de contenido del instrumento en cuestión, es decir, determinar si el instrumento estaba midiendo aspectos acordes con los objetivos propuestos y saber si constituía una muestra adecuada y representativa de los elementos a considerar para cada uno de los indicadores, se contó con el juicio de expertos. 
Los expertos valoraron la pertinencia y claridad de los elementos en una escala aditivo global tipo Likert de 6 opciones de respuesta (siendo 1 el mínimo y 6 el máximo). Asimismo, valoraron su conformidad con la agrupación de los ítems en los diferentes indicadores.

Una vez elaborada la base de datos, se calculó la fiabilidad de las respuestas emitidas por los expertos, relativas a la pertinencia de los ítems mediante el estadístico alpha de Cronbach. Este coeficiente nos permite cuantificar el nivel de fiabilidad de las respuestas a una escala de medida como la que nos ocupa considerando la magnitud inobservable construida a partir de las n variables observadas partiendo de las respuestas de los jueces. Se halló un valor de 0,897. Mientras que para la claridad el resultado del análisis fue de 0,907. Hay que advertir que el nivel máximo de correlación es igual a 1. Si los ítems fueran independientes entre sí, entonces el valor sería 0. Los resultados hallados evidencian una alta coherencia interna de las respuestas emitidas por los jueces expertos ya que se considera que existe una buena consistencia interna cuando el valor de alfa es superior a $0,7$.

Del mismo modo, el análisis de validez de contenido basado en el modelo matemático Lawshe (1975, en Cohen y Swerdlik, 2006) tomó en consideración la tabla de valores mínimos de razón para un total de 20 jueces y el índice de validez de contenido mínimo para ser aceptado resultó ser de 0,42. De tal modo, todos los ítems que obtuvieron menor puntuación fueron rechazados.

Asimismo, se consideró modificar aquellos ítems con puntuaciones medias en claridad iguales o inferiores a 4,5. Del mismo modo, se tomó en consideración el conjunto de las aportaciones cualitativas de los jueces al instrumento para reformular aquellos ítems que no resultaron suficientemente claros. En consecuencia, se reformularon dos ítems, se reubicó un elemento dentro de otro indicador y dos ítems dieron lugar a un solo elemento. Igualmente, se cambió el nombre a uno de los indicadores a petición de ocho expertos.

Finalmente, el resultado de esta fase se muestra resumido en la tabla I.

Como se puede observar, el resultado de esta tercera fase de estudio permitió seleccionar 57 de los 70 ítems iniciales. A continuación, se detalla la información relativa a los ítems del cuestionario piloto que han sido eliminados, modificados o reubicados en el cuestionario definitivo (tabla II).

Finalmente, el instrumento definitivo, que denominamos QuaSciweb, se presenta en la tabla III.

Se recomienda aplicar este instrumento únicamente para su uso previsto en evaluación de portales y webs de ámbito científico. Esta herramienta proporciona datos que verifican la existencia de ítems de calidad estándar en estos alojamientos. Para promover un mejor servicio se recomienda una utilización anticipada al lanzamiento del portal o web en el servidor. Los profesionales que lo utilicen deben formularse a modo de pregunta sencilla si la web o portal posee cada uno de los ítems planteados. De tal modo, las respuestas serán sí o no y se irán confirmando y descartando paulatinamente cada uno de los elementos. Algunas webs y portales requerirán de evaluaciones adicionales por parte de proveedores y diseñadores web. 


\section{TABLA I}

Resultados del análisis estadístico

\begin{tabular}{|c|c|c|c|}
\hline \multicolumn{4}{|c|}{ 1. IDENTIDAD Y AUTORÍA } \\
\hline Ítems & $\begin{array}{c}\text { Media } \\
\text { claridad }\end{array}$ & $\begin{array}{c}\text { Media } \\
\text { pertinencia }\end{array}$ & CVR \\
\hline $\begin{array}{l}1.1 \\
1.2 \\
1.3 \\
1.4 \\
1.5 \\
1.6 \\
\end{array}$ & $\begin{array}{l}5,5 \\
5,05 \\
\mathbf{4 , 3 8}, \mathbf{3 8 * * *} \\
4,12 \\
4,77 \\
4,75\end{array}$ & $\begin{array}{l}5,5 \\
4,77 \\
4,17 \\
4,35 \\
4,94 \\
4,31 \\
\end{array}$ & $\begin{array}{l}1 \\
0,88 \\
0,66 \\
\mathbf{0 , 2 9} * \\
0,76 \\
\mathbf{0 , 3 7 *} \\
\end{array}$ \\
\hline \multicolumn{4}{|c|}{ 2. INTERFAZ DE USUARIO } \\
\hline Ítems & $\begin{array}{c}\text { Media } \\
\text { claridad }\end{array}$ & $\begin{array}{c}\text { Media } \\
\text { pertinencia }\end{array}$ & CVR \\
\hline $\begin{array}{l}2.1 \\
2.2 \\
2.3 \\
2.4 \\
2.5 \\
2.6 \\
2.7 \\
2.8 \\
2.9 \\
2.10 \\
2.11 \\
2.12 \\
2.13 \\
2.14 \\
2.15 \\
2.16 \\
2.17 \\
2.18 \\
\end{array}$ & $\begin{array}{l}5,28 \\
5,05 \\
4,88 \\
4,88 \\
4,66 \\
3,94 \\
5,25 \\
5,44 \\
5,38 \\
5,61 \\
5,44 \\
5,25 \\
4,87 \\
4,83 \\
5,36 \\
5,12 \\
5,22 \\
4,77\end{array}$ & $\begin{array}{l}5,38 \\
5,22 \\
5,27 \\
4,12 \\
4,5 \\
5,19 \\
5,37 \\
5,66 \\
5,61 \\
5,55 \\
4,94 \\
5,37 \\
4,5 \\
5,22 \\
5,28 \\
5,25 \\
5,27 \\
3,33 \\
\end{array}$ & $\begin{array}{l}0,88 \\
0,77 \\
1 \\
\mathbf{0 , 0 5} \\
\mathbf{0 , 5} 5^{* *} \\
\mathbf{0 , 7 6 * *} \\
0,64 \\
0,76 \\
0,76 \\
1 \\
1 \\
0,88 \\
\mathbf{0 , 4 1 *} \\
1 \\
0,88 \\
0,76 \\
0,88 \\
\mathbf{0}^{*} \\
\end{array}$ \\
\hline \multicolumn{4}{|c|}{ 3. CONTENIDO } \\
\hline Ítems & $\begin{array}{c}\text { Media } \\
\text { claridad }\end{array}$ & $\begin{array}{c}\text { Media } \\
\text { pertinencia }\end{array}$ & CVR \\
\hline $\begin{array}{l}3.1 \\
3.2 \\
3.3 \\
3.4 \\
3.5 \\
3.6 \\
3.7 \\
3.8\end{array}$ & $\begin{array}{l}4,66 \\
5,38 \\
5,81 \\
5,38 \\
5 \\
4,83 \\
5,72 \\
5\end{array}$ & $\begin{array}{l}5,39 \\
5,44 \\
5,5 \\
5,22 \\
5,17 \\
5,22 \\
5,3 \\
4,44\end{array}$ & $\begin{array}{l}\mathbf{0 , 3 3 *} \\
1 \\
0,87 \\
0,88 \\
0,64 \\
0,88 \\
1 \\
\mathbf{0 , 3 8 *}\end{array}$ \\
\hline
\end{tabular}

4. NAVEGACIÓN Y RECUPERACIÓN DE DATOS

\begin{tabular}{l|l|c|l}
\hline \multicolumn{1}{c|}{ Ítems } & $\begin{array}{c}\text { Media } \\
\text { claridad }\end{array}$ & $\begin{array}{l}\text { Media per- } \\
\text { tinencia }\end{array}$ & CVR \\
\hline 4.1 & 5,27 & 5,44 & 0,88 \\
4.2 & 4 & 4,2 & $\mathbf{0 , 4 1 *}$ \\
4.3 & 4,66 & 5,19 & 1 \\
4.4 & 5,61 & 5,61 & 1 \\
4.5 & 5,22 & 5,44 & 0,88 \\
4.6 & 4,83 & 5 & 0,88 \\
4.7 & 5 & 5,41 & 0,77 \\
4.8 & 5,55 & 5,61 & 1 \\
4.9 & 5,33 & 5,38 & 1 \\
4.10 & 5,06 & 4,87 & 0,75 \\
4.11 & 5,05 & 5 & 0,66 \\
\hline
\end{tabular}

\begin{tabular}{|c|c|c|c|}
\hline \multicolumn{4}{|c|}{ 5. EXPERIENCIA DE USUARIO } \\
\hline Ítems & $\begin{array}{l}\text { Media } \\
\text { claridad }\end{array}$ & $\begin{array}{c}\text { Media } \\
\text { pertinencia }\end{array}$ & CVR \\
\hline 5.1 & 4,35 & 4,52 & $0,40 *$ \\
\hline 5.2 & 4,88 & 5,06 & 0,75 \\
\hline 5.3 & 5,22 & 5,29 & 0,81 \\
\hline 5.4 & 5,33 & 5,11 & 0,76 \\
\hline 5.5 & 5,16 & 4,52 & $0,40 *$ \\
\hline 5.6 & 5,16 & 5,33 & 0,88 \\
\hline 5.7 & 5,66 & 5,53 & 1 \\
\hline 5.8 & 5,27 & 4,5 & $\mathbf{0 , 3 3 *}$ \\
\hline
\end{tabular}

\begin{tabular}{l|l|c|l}
\hline Ítems & $\begin{array}{c}\text { Media } \\
\text { claridad }\end{array}$ & $\begin{array}{c}\text { Media } \\
\text { pertinencia }\end{array}$ & CVR \\
\hline 6.1 & 5,33 & 5,22 & 0,77 \\
6.2 & 5,53 & 5,44 & 1 \\
6.3 & $\mathbf{4 , 5 * * * *}$ & 5,31 & 1 \\
6.4 & 5,38 & 5,33 & 1 \\
6.5 & 4,72 & 5,05 & 0,77 \\
6.6 & 5,05 & 5,06 & 0,77 \\
6.7 & 5,29 & 5,41 & 0,77 \\
6.8 & 5,83 & 5,88 & 1 \\
6.9 & 5,72 & 5,55 & 1 \\
6.10 & 4,94 & 4,94 & 0,66 \\
6.11 & 5 & 6,12 & 0,66 \\
6.12 & 5,38 & 5,44 & 1 \\
6.13 & 5,27 & 4,83 & $\mathbf{0 , 6 6 * * *}$ \\
6.14 & 5,16 & 4,83 & 0,66 \\
6.15 & 4,94 & 4,1 & $\mathbf{0 , 4 1 *}$ \\
6.16 & 5,5 & 5,75 & 1 \\
6.17 & 4,94 & 5,33 & 0,77 \\
6.18 & 5,22 & 5,05 & 0,88 \\
6.19 & 5,05 & 4,81 & 0,62 \\
\hline
\end{tabular}

* Ítems eliminados tomando como criterio el CVR inferior a 0,42. ** Ítems adaptados que se han unido en

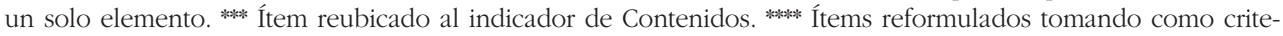
rio la claridad. 
TABLA II

Información detallada de los elementos del cuestionario piloto que han sido eliminados, modificados o reubicados en el cuestionario definitivo

\begin{tabular}{|c|c|}
\hline 1. Identidad y autoría & Acciones realizadas \\
\hline $\begin{array}{l}\text { 1.3. Presenta la historia del organismo autor } \\
\text { de la web. }\end{array}$ & $\begin{array}{l}\text { Ítem reformulado tomando como criterio el } \\
\text { valor obtenido en claridad. Se modifica in- } \\
\text { cluyendo "propósito de la web". }\end{array}$ \\
\hline 1.4. Existencia de código de ética. & $\begin{array}{l}\text { Ítem eliminado tomando como criterio el } C V R \\
\text { inferior a } 0,42 \text {. }\end{array}$ \\
\hline $\begin{array}{l}\text { 1.6. Prestigio y reconocimiento de la institu- } \\
\text { ción. }\end{array}$ & $\begin{array}{l}\text { Ítem eliminado tomando como criterio el } C V R \\
\quad \text { inferior a } 0,42 \text {. }\end{array}$ \\
\hline 2. Interfaz de usuario & Acciones realizadas \\
\hline 2.4. Existencia de un foro de discusión. & $\begin{array}{l}\text { Ítem eliminado tomando como criterio el } C V R \\
\quad \text { inferior a } 0,42 \text {. }\end{array}$ \\
\hline 2.5. Existencia de un blog. & $\begin{array}{l}\text { Ítem adaptado que se ha unido junto al } 2.6 \text { en } \\
\text { un solo elemento. Se reformula en el si- } \\
\text { guiente ítem: "Existencia de espacios interac- } \\
\text { tivos". }\end{array}$ \\
\hline $\begin{array}{l}\text { 2.6. Existencia de foro y espacios colabora- } \\
\text { tivos. }\end{array}$ & $\begin{array}{l}\text { Ítem adaptado que se ha unido junto al } 2.5 \text { en } \\
\text { un solo elemento. Se reformula en el si- } \\
\text { guiente ítem: "Existencia de espacios interac- } \\
\text { tivos". }\end{array}$ \\
\hline 2.13. Visibilidad de usuarios conectados. & $\begin{array}{l}\text { Ítem eliminado tomando como criterio el } C V R \\
\text { inferior a } 0,42 \text {. }\end{array}$ \\
\hline $\begin{array}{l}\text { 2.18. Contempla la posibilidad de realizar } \\
\text { compras de artículos, materiales o recursos } \\
\text { integrando uno/varios sistemas de pago (pa- } \\
\text { ypal, pago a través de tarjeta de crédi- } \\
\text { to, etc.). }\end{array}$ & $\begin{array}{l}\text { Ítem eliminado tomando como criterio el } C V R \\
\text { inferior a } 0,42 \text {. }\end{array}$ \\
\hline 3. Contenido & Acciones realizadas \\
\hline $\begin{array}{l}\text { 3.1. Prestigio y calidad de las fuentes (exis- } \\
\text { tencia de información valiosa y riguro- } \\
\text { sa, etc.). }\end{array}$ & $\begin{array}{l}\text { Ítem eliminado tomando como criterio el } C V R \\
\text { inferior a } 0,42 \text {. Respecto a este ítem, uno de } \\
\text { los comentarios más señalados por los jue- } \\
\text { ces fue: "la dificultad de valorar objetiva- } \\
\text { mente este item». }\end{array}$ \\
\hline $\begin{array}{l}\text { 3.8. La información que aparece en la web } \\
\text { tiene cierto grado de objetividad determina- } \\
\text { da si se observan hechos o de datos. }\end{array}$ & $\begin{array}{l}\text { Ítem eliminado tomando como criterio el } C V R \\
\text { inferior a } 0,42 \text {. }\end{array}$ \\
\hline 4. Navegación y recuperación de datos & Acciones realizadas \\
\hline $\begin{array}{l}\text { 4.2. Se encuentran orientaciones de contexto } \\
\text { cuando pasamos el cursor por encima de } \\
\text { texto y/o imagen. }\end{array}$ & $\begin{array}{l}\text { Ítem eliminado tomando como criterio el } C V R \\
\text { inferior a } 0,2 \text {. }\end{array}$ \\
\hline
\end{tabular}


TABLA II (continuación)

\begin{tabular}{|c|c|}
\hline 5. Experiencia de usuario & Acciones realizadas \\
\hline 5.1. Buena relación figura-fondo. & $\begin{array}{l}\text { Ítem eliminado tomando como criterio el } C V R \\
\text { inferior a } 0,42 \text {. Respecto a este ítem, uno de } \\
\text { los comentarios más señalados por los jue- } \\
\text { ces fue: "Se trata de un elemento demasiado } \\
\text { subjetivo para ser tomado en consideración". }\end{array}$ \\
\hline $\begin{array}{l}\text { 5.5. Distribución espacial de la página no } \\
\text { saturada. Se trata de un elemento demasia- } \\
\text { do subjetivo para ser tomado en considera- } \\
\text { ción. }\end{array}$ & $\begin{array}{l}\text { Ítem eliminado tomando como criterio el } C V R \\
\quad \text { inferior a } 0,42 \text {. }\end{array}$ \\
\hline 5.8. Presencia de metaetiquetas básicas. & $\begin{array}{l}\text { Ítem eliminado tomando como criterio el } C V R \\
\text { inferior a } 0,42 \text {. }\end{array}$ \\
\hline 6. Visibilidad y divulgación & Acciones realizadas \\
\hline 6.3. Presencia de enlaces evaluados. & $\begin{array}{l}\text { Îtems reformulados tomando como criterio la } \\
\text { claridad. Los jueces solicitaron que se con- } \\
\text { cretase más este aspecto. Quedó reformula- } \\
\text { do del siguiente modo: «Enlaces evaluados } \\
\text { y seleccionados en relación con los conteni- } \\
\text { dos de la web. }\end{array}$ \\
\hline 6.13. Existencia de boletín y noticias. & Ítem reubicado al indicador de Contenidos. \\
\hline $\begin{array}{l}\text { 6.15. La web ofrece servicios de consultoría } \\
\text { y gestión de empresas. }\end{array}$ & $\begin{array}{l}\text { Ítem eliminado tomando como criterio el } C V R \\
\text { inferior a } 0,42 \text {. }\end{array}$ \\
\hline
\end{tabular}

TABLA III

Instrumento de verificación "QuaSciWeb"

\begin{tabular}{l|c|c}
\hline \multicolumn{1}{c}{ 1. IdSTA DE VERIFICACIÓN } & Sí & No \\
\hline Incluye datos de contacto. & & \\
\hline Especifica el ámbito temático y usuarios. & & \\
\hline Historia y propósito. & & \\
\hline Autoría del sitio web determinada. & & \\
\hline Número de elementos que cumple & & \\
\hline \multicolumn{1}{c|}{$\mathbf{2 .}$ Interfaz de usuario } & Sí & No \\
\hline Localización funcional de los menús (lateral izquierda o superior). & & \\
\hline Posibilidad de contactar con el autor, institución o webmaster & & \\
\hline Presencia de buscador de red o metabuscadores. & & \\
\hline Existencia de espacios interactivos. & & \\
\hline Lista de distribución de correo. & & \\
\hline
\end{tabular}


TABLA III (continuación)

\section{LISTA DE VERIFICACIÓN}

\begin{tabular}{|c|c|c|}
\hline 2. Interfaz de usuario & Sí & No \\
\hline \multicolumn{3}{|l|}{ Acceso controlado y registro. } \\
\hline \multicolumn{3}{|l|}{ Existe servicio para el depósito de ficheros y direcciones. } \\
\hline \multicolumn{3}{|l|}{ Calendario de eventos y actividades } \\
\hline \multicolumn{3}{|l|}{ Dispone de un apartado de FAQ. } \\
\hline \multicolumn{3}{|l|}{ Arquitectura de la información (existencia de mapa de la web). } \\
\hline \multicolumn{3}{|l|}{ Inclusión de redes sociales. } \\
\hline \multicolumn{3}{|l|}{ Posibilidad de suscripción a servicios de información de contenidos de la propia web. } \\
\hline \multicolumn{3}{|l|}{ Existencia de rankings de visitas y descargas. } \\
\hline \multicolumn{3}{|l|}{ Presencia de un menú de ayuda. } \\
\hline \multicolumn{3}{|l|}{ Número de elementos que cumple } \\
\hline 3. Contenido & Sí & No \\
\hline \multicolumn{3}{|l|}{ Gramática, ortografía y sintaxis correcta en sus informaciones. } \\
\hline \multicolumn{3}{|l|}{ Volumen de información suficiente (extensión, coherencia) } \\
\hline \multicolumn{3}{|l|}{ Información contrastada y rigurosa (editada, supervisada). } \\
\hline \multicolumn{3}{|l|}{ Grandes áreas de distribución de contenidos. } \\
\hline \multicolumn{3}{|l|}{ Información actualizada y mantenimiento de los contenidos. } \\
\hline \multicolumn{3}{|l|}{ Presenta contenidos elaborados expresamente para la web. } \\
\hline \multicolumn{3}{|l|}{ Existencia de boletín y noticias. } \\
\hline \multicolumn{3}{|l|}{ Número de elementos que cumple } \\
\hline 4. Navegación y recuperación de datos & Sí & No \\
\hline \multicolumn{3}{|l|}{ Navegación intuitiva. } \\
\hline \multicolumn{3}{|l|}{ Jerarquización de contenidos. } \\
\hline \multicolumn{3}{|l|}{ Sistema de búsqueda sobre contenidos de la propia web. } \\
\hline \multicolumn{3}{|l|}{ Sumarios locales o secciones (ej. resúmenes previo a descargas). } \\
\hline \multicolumn{3}{|l|}{ Índices temáticos, cronológicos y/o geográficos. } \\
\hline \multicolumn{3}{|l|}{ Navegación semántica. } \\
\hline \multicolumn{3}{|l|}{ Economía de tiempos en la navegación. } \\
\hline \multicolumn{3}{|l|}{ Iconos de navegación y leyendas. } \\
\hline \multicolumn{3}{|l|}{ Indicadores de números de archivos } \\
\hline \multicolumn{3}{|l|}{ Permite acceso a dispositivos móviles. } \\
\hline \multicolumn{3}{|l|}{ Número de elementos que cumple } \\
\hline 5. Experiencia de usuario & Sí & No \\
\hline \multicolumn{3}{|l|}{ Tipografía adecuada y adaptada. } \\
\hline Márgenes a ambos lados del texto y entre párrafos. & & \\
\hline
\end{tabular}


TABLA III (continuación)

\begin{tabular}{|c|c|c|}
\hline \multicolumn{3}{|l|}{ LISTA DE VERIFICACIÓN } \\
\hline 5. Experiencia de usuario & Sí & No \\
\hline \multicolumn{3}{|l|}{$\begin{array}{l}\text { Presencia de elementos estáticos, dinámicos y multimedia que completan la infor- } \\
\text { mación. }\end{array}$} \\
\hline \multicolumn{3}{|l|}{ Visualización agradable (apariencia de limpieza). } \\
\hline \multicolumn{3}{|l|}{ Paleta de colores adecuada a la imagen de la institución. } \\
\hline \multicolumn{3}{|l|}{ Número de elementos que cumple } \\
\hline 6. Visibilidad y divulgación & Sí & No \\
\hline \multicolumn{3}{|l|}{ Enlaces a fuentes actualizadas } \\
\hline \multicolumn{3}{|l|}{$\begin{array}{l}\text { Facilita el acceso a personas con discapacidad, al menos, con un nivel de confor- } \\
\text { midad «Doble A». }\end{array}$} \\
\hline \multicolumn{3}{|l|}{ Enlaces evaluados y seleccionados en relación con los contenidos de la web. } \\
\hline \multicolumn{3}{|l|}{ Enlaces a bases de datos (catálogos, bibliotecas y repositorios). } \\
\hline \multicolumn{3}{|l|}{ Título del web en la barra del navegador. } \\
\hline \multicolumn{3}{|l|}{ Icono corporativo en la barra del navegador. } \\
\hline \multicolumn{3}{|l|}{ Contenido de los archivos en los primeros párrafos y posibilidad de "leer más». } \\
\hline \multicolumn{3}{|l|}{ Cumple los requisitos estándares W3C para visualización en diversos navegadores. } \\
\hline \multicolumn{3}{|l|}{ Carga imágenes rápidamente } \\
\hline \multicolumn{3}{|l|}{ Enlaces desde otras páginas. } \\
\hline \multicolumn{3}{|l|}{$\begin{array}{l}\text { La web está avalada por una empresa o institución de reconocido prestigio — no } \\
\text { confundir con publicidad puntual—. }\end{array}$} \\
\hline \multicolumn{3}{|l|}{ Incluye fecha de última actualización } \\
\hline \multicolumn{3}{|l|}{ Visibilidad de las condiciones de uso y/o términos legales } \\
\hline \multicolumn{3}{|l|}{ Difusión en dos o más idiomas. } \\
\hline \multicolumn{3}{|l|}{ Incluye algún tipo de índice de posicionamiento en buscadores. } \\
\hline \multicolumn{3}{|l|}{ Número de elementos que cumple } \\
\hline Total de elementos que cumple & & \\
\hline
\end{tabular}

Como se ha indicado, el QuaSciWeb es una herramienta de evaluación fácil de usar destinada a valorar a varios indicadores de calidad. El análisis de cada uno de los mismos es independiente. De tal modo, cada uno de ellos recogerá una serie de ítems y determinará el cumplimiento de esos requisitos para ese indicador independientemente del resto. Así pues, la valoración definitiva se ha de realizar sobre la base de los seis indicadores y se podrá obtener una puntuación total orientativa no ponderada. 


\section{Conclusiones}

El procedimiento seguido ha sido riguroso, atendiendo a un método combinado en el que se ha tenido en cuenta: el análisis de documentos, las valoraciones cualitativas de los jueces y las valoraciones cuantitativas. Tras una revisión exhaustiva de la literatura científica no se hallaron evidencias de estudios metodológicos similares para construir un instrumento que permita determinar la calidad de portales y webs de carácter científico.

En definitiva, tras una revisión final del proceso cabe señalar que los jueces valoraron el cuestionario y la temática de investigación de manera favorable. Sus consideraciones contribuyeron a mejorar la validez de contenido del instrumento dado que permitieron depurar los diferentes elementos e indicadores. Asimismo, se ha de tener en cuenta que para evitar que se produjera cualquier tipo de influencia en la revisión entre los expertos, los documentos fueron enviados de manera independiente a lo largo del segundo semestre de 2010.

El instrumento definitivo QuaSciWeb puede ser utilizado con tres objetivos: 1) valorar la calidad de portales y páginas web que ofrezcan contenidos de investigación de carácter científico, 2) identificar los contenidos necesarios para su mejora y 3) proveer de unas directrices concretas a tener en cuenta por los programadores y diseñadores web a la hora de construir una página o un portal de estas características.

\section{Agradecimientos}

Esta investigación ha sido financiada por el Plan Nacional I+D+i del Ministerio de Ciencia e Innovación con la referencia DEP2010-12214-E y por el Consejo Superior de Deportes.

\section{Bibliografía}

Aguillo, I. F., Granadino, B., Ortega, J. L.; Prieto, J. A. (2006): Scientific research activity and communication measured with cybermetrics indicators. Journal of the American Society for Information Science and Technology, vol. 57 (10), 1296-1302.

Alexa Internet (2011): The web information company. http://www.alexa.com [consultado el 26 de julio de 2011].

Almind, T. C.; Ingwersen, P. (1997): Informetric analyses on the world wide web: Methodological approaches to "webometrics". Journal of Documentation, vol. 53 (4), 404-426.

Alonso Berrocal, J. L.; García Figuerola, C.; Zazo, Á. F. (2008): Recuperación de información web: 10 años de cibermetría. Ibersid: revista de sistemas de información y documentación, vol. 2, 69-78.

Arakaki, M.; Willett, P. (2009): Webometric analysis of departments of librarianship and information science: A follow-up study. Journal of Information Science, vol. 35 (2), 143-142. 
Arroyo, N.; Ortega, J. L.; Pareja, V.; Prieto, J. A.; Aguillo, I. (2005): Cibermetría. Estado de la cuestión. Actas de las IX Jornadas Españolas de Documentación FESABID. Madrid, España: FESABID. http://digital.csic.es/bitstream/10261/4296/1/R-17.pdf [consultado el 26 de julio de 2011].

Barjak, F.; Thelwall, M. (2008): A statistical analysis of the web presences of European life sciences research teams. Journal of the American Society for Information Science and Technology, vol. 59 (4), 628-643.

Barnes, S. J.; Vidgen, R. T. (2002): An integrative approach to the assessment of e-commerce quality. Journal of Electronic Commerce Research, vol. 3 (3), 114-127.

Batlle-Montserrat, J.; Abadal, E.; Blat, J. (2011): Benchmarking del e-gobierno local: Limitaciones de los métodos de evaluación comparativa. El Profesional de la Información, vol. 20 (3), 251-259.

Björneborn, L.; Ingwersen, P. (2001): Perspective of webometrics. Scientometrics, vol. 50 (1), 65-82.

Björneborn, L.; Ingwersen, P. (2004): Toward a basic framework for webometrics. Journal of the American Society for Information Science and Technology, vol. 55 (14), 12161227.

Caraballo-Pérez, Y.; Torres-Cárdenas, V.; Noda-Amoros, A.-C.;Herrera-Toscano, J. A. (2008): Medidas de popularidad de la educación superior cubana: Un análisis webmétrico. El Profesional de la Información, vol. 17 (4), 44-448.

Chao, H. Y. (2002): Assessing the quality of academic libraries on the Web: The development and testing of criteria. Library \& Information Science Research, vol. 24 (2), 169-194.

Choudrie, J.; Ghinea, G.; Weerakkody, V. (2004): Evaluating global e-government sites: A view using web diagnostic tools. Electronic Journal of e-Government, vol. 2 (2), 105-114.

Codina, L.;Marcos, M. C. (2005): Posicionamiento web: conceptos y herramientas. El Profesional de la Información, vol. 14 (2), 84-99.

Cohen, R. J.;Swerdlik, M. E. (2006): Pruebas y evaluación psicológicas. Introducción a las pruebas y a la medición. México D.F.; McGraw-Hill, 807 p.

DeVon, H. A.; Block, M. E.; Oyle-Wright, P.; Ernst, D. M.; Hayden, S. J.; Lazzara, D. J.; Savoy, S. M.; Kostas-Polston, E. (2007): A psychometric toolbox for testing validity and reliability. Journal of Nursing Scholarship, vol. 39 (2), 155-164.

Diniz, E.; Morena Porto, R.; Adachi, T. (2005): Internet banking in Brazil: Evaluation of functionality, reliability and usability. The Electronic Journal Information Systems Evaluation, vol. 8 (1), 41-50.

González-Lucio, J. A.; Faba-Pérez, C.; de Moya Anegón, F.; Moscoso-Castro, P. (2009): Evolution of the formal quality indicators of the Web spaces of University Libraries in Spain. Cybermetrics, vol. 13 (1), paper 1.

Hasan, L.; Abuelrub, E. (2011): Assessing the quality of web sites. Applied Computing and Informatics, vol. 9 (1), 11-29.

Hussin, H.; Suhaimi, M. A.; Mustafa, M. (2005): E-Commerce and ethical web design: Applying the BBBOnline guidelines on Malaysian web sites. The International Arab Journal of Information Technology, vol. 2 (3), 219-227. 
Ingwersen, P. (1998): The calculation of Web impact factors. Journal of Documentation, vol. 54 (2), 236-243.

Lautenbach, M. A. E.; Schegget, I. S.; Schoute, A. M.; Witteman, C. L. M. (2006): Evaluating the usability of web pages: A case study: Utrecht University. http://www.phil.uu.nl/ preprints/ckipreprints/PREPRINTS/preprint011.pdf [consultado el 26 de julio de 2011].

Lynn, M. R. (1986): Determination and quantification of content validity. Nursing Research, vol. 35, 382-385.

Marcos, M. C. (2006): Webometrics pone orden en las universidades. El Profesional de la Información, vol. 15 (3), 231-236.

Maynard, M.; Tian, Y. (2004): Between global and glocal: content analysis of the Chinese Web Sites of the 100 top global brands. Public Relations Review, vol. 30 (3), 285-291.

Mebrate, T. W. (2010): A framework for evaluating academic website quality from students perspective. Tesis de Master no publicada. Delft University of Technology.

Millman, J.; Greene, J. (1989): The specification and development of test of achievement and ability. En: Linn, R. (editor) Educational Measurement. Macmillan, London, UK.

Miranda, F. J.; Cortés, R.; Barriuso, C. (2006): Quantitative evaluation of e-banking web sites: An empirical study of Spanish banks. The Electronic Journal Information Systems Evaluation, vol. 9 (2), 73-82.

Monaghan, B. (2008): The limitations of eGovernment evaluation: A critical review. iSCHANNEL, vol. 3 (1), 16-19.

Muñiz, José; Fidalgo, A.M.; Cueto, E.G.; Martínez, R.; Moreno, R. (2005): Análisis de los ítems. Madrid: La Muralla, p. 181.

Olsina, L.; Rossi, G. (2002): Measuring Web application quality with WebQEM. Ieee Multimedia, vol. 9 (4), 20-29.

Ortega, J. L.; Aguillo, I. (2006): Análisis de co-enlaces: una aproximación teórica. El Profesional de la Información, vol. 15 (4), 270-277.

Ortega, J. L.; Aguillo, I. (2007): La web académica española en el contexto del Espacio Europeo de Educación Superior: Estudio exploratorio. El Profesional de la Información, vol. 16 (5), 417-425.

Ortega, J. L.; Aguillo, I. (2009): Análisis estructural de la web académica iberoamericana. Revista Española de Documentación Científica, 32 (3), 51-65.

Ortega, J. L.; Aguillo, I.; Prieto, J. A. (2006): Longitudinal study of contents and elements in the scientific web environment. Journal of Information Science, vol. 32 (4), 344-351.

Page, L.; Brin, S.; Motwani, R.; Winograd, T. (1998): The PageRank citation ranking: Bringing order to the web. Technical report: Stanford Digital Library Technologies Project.

Palatano, M. (2002): Análisis de los sitios web de las bibliotecas universitarias argentinas. El Profesional de la Información, vol. 11 (2), 102-110.

Pinto, M.; Alonso Berrocal, J. L.; Cordón García, J. A.; Fernández Marcial, V.; García Figuerola, C.; García Marco, J. (2005): Quality assessment of Spanish universities' Web sites focused on the European Research Area. Scientometrics, vol. 65 (1), 67-93.

Pinto, M.; Alonso Berrocal, J. L.; Cordón García, J. A.; Fernández Marcial, V.; García Figuerola, C.; García Marco, J. (2004): Análisis cualitativo de la visibilidad de la investigación de las universidades españolas a través de sus páginas web. Revista Española de Documentación Científica, vol. 27 (3), 345-370. 
Pinto, M.; Guerrero, D.; Fernandez-Ramos, A.; Doucet, A. V. (2009): Information provided by Spanish university websites on their assessment and quality processes. Scientometrics, vol. 81 (1), 265-289.

Raman, M.; Stephenaus, R.; Alam, N.; Kuppusamy, M. (2008): Information technology in Malaysia: E-service quality and uptake of internet banking. Journal of Internet Banking and Commerce, vol. 13 (2).

Ribera, M.; Térmens, M.; Frías, A. (2009): La accessibilidad de las webs de las universidades españolas. Balance 2001-2006. Revista Española de Documentación Científica, vol. 32 (3), 66-88.

Rousseau, R. (1997): Sitations: An exploratory study. Cybermetrics, vol. 1 (1), paper 1.

Rovira, C. F.; Marcos, M. C.; Codina, L. (2007): Repositorios de publicaciones digitales de libre acceso en Europa: Análisis y valoración de la accesibilidad, posicionamiento web y calidad del código. El Profesional de la Información, vol. 16 (1), 24-38.

Smith, A. G. (1999): The impact of web sites: A comparison between Australasia and Latin America. Actas del Congreso Internacional de Información, INFO'99. La Habana, Cuba. http://www.vuw.ac.nz/staff/alastair_smith/publns/austlat [consultado el 26 de julio de 2011].

Thelwall, M. (2008): Bibliometrics to webometrics. Journal of Information Science, vol. 34 (4), 605-621.

Thelwall, M.; Harries, G. (2003): The connection between the research of a university and counts of links to its web pages: An investigation based upon a classification of the relationships of pages to the research of the host university. Journal of the American Society for Information Science and Technology, vol. 54 (7), 594-602.

Thelwall, M.; Sud, P. (2011): A comparison of methods for collecting web citation data for academic organizations. Journal of the American Society for Information Science and Technology, vol. 62 (8), 1488-1497.

Thelwall, M.; Vaugan, L.; Björneborn, L. (2005): Webometrics. Annual Review of Information Science and Technology, vol. 35, 81-135.

Thomas, O.; Willett, P. (2000): Webometric analysis of departments of librarianship and information science. Journal of Information Science, vol. 26 (6), 421-428.

Wyman, S. K.; Beachboard, J. C.; McClure, C. R. (1997): User and system-based quality criteria for evaluating information resources and services available from federal websites: Final report: Syracuse University. http://eric.ed.gov/ERICWebPortal/search/detailmini.jsp?_nfpb=true\&_\&ERICExtSearch_SearchValue_0=ED409020\&ERICExtSearch_ SearchType_0=no\&accno=ED 409020 [consultado el 26 de julio de 2011]. 